\title{
Prevalence and patterns of cooking dinner at home in the USA: National Health and Nutrition Examination Survey (NHANES) 2007-2008
}

\author{
Senbagam Virudachalam ${ }^{1, *}$, Judith A Long ${ }^{2,3,4}$, Michael O Harhay ${ }^{2}$, Daniel E Polsky ${ }^{2,4}$ \\ and Chris Feudtner ${ }^{1,4,5}$ \\ 'Division of General Pediatrics, The Children's Hospital of Philadelphia, 34th St. \& Civic Center Blvd, CHOP \\ North Room 1416, Philadelphia, PA 19104, USA: ${ }^{2}$ Division of General Internal Medicine, Perelman School of \\ Medicine, University of Pennsylvania, Philadelphia, PA, USA: ${ }^{3}$ Philadelphia VA Center for Health Equity \\ Research and Promotion, Philadelphia, PA, USA: ${ }^{4}$ Leonard Davis Institute of Health Economics, University of \\ Pennsylvania, Philadelphia, PA, USA: ${ }^{5}$ Department of Pediatrics, Perelman School of Medicine, University of \\ Pennsylvania, Philadelphia, PA, USA
}

Submitted 31 January 2013: Final revision received 17 June 2013: Accepted 7 August 2013: First published online 10 0ctober 2013

\begin{abstract}
Objective: To measure the prevalence of cooking dinner at home in the USA and test whether home dinner preparation habits are associated with socio-economic status, race/ethnicity, country of birth and family structure.

Design: Cross-sectional analysis. The primary outcome, self-reported frequency of cooking dinner at home, was divided into three categories: 0-1 dinners cooked per week ('never'), 2-5 ('sometimes') and 6-7 ('always'). We used bivariable and multivariable regression analyses to test for associations between frequency of cooking dinner at home and factors of interest.

Setting: The 2007-2008 National Health and Nutrition Examination Survey (NHANES).

Subjects: The sample consisted of 10149 participants.

Results: Americans reported cooking an average of five dinners per week; $8 \%$ never, 43\% sometimes and 49\% always cooked dinner at home. Lower household wealth and educational attainment were associated with a higher likelihood of either always or never cooking dinner at home, whereas wealthier, more educated households were more likely to sometimes cook dinner at home $(P<0 \cdot 05)$. Black households cooked the fewest dinners at home (mean $=4 \cdot 4$, $95 \%$ CI $4 \cdot 2,4 \cdot 6$ ). Households with foreign-born reference persons cooked more dinners at home $($ mean $=5 \cdot 8,95 \%$ CI $5 \cdot 7,6 \cdot 0)$ than households with US-born reference persons $($ mean $=4 \cdot 9,95 \% \mathrm{CI} 4 \cdot 7,5 \cdot 1)$. Households with dependants cooked more dinners at home (mean $=5 \cdot 2,95 \%$ CI $5 \cdot 1,5 \cdot 4)$ than households without dependants $($ mean $=4 \cdot 6,95 \%$ CI 4·3, 5•0).

Conclusions: Home dinner preparation habits varied substantially with socioeconomic status and race/ethnicity, associations that likely will have implications for designing and appropriately tailoring interventions to improve home food preparation practices and promote healthy eating.
\end{abstract}

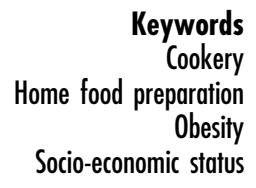

Preparing food at home is a complex behaviour entailing a sequence of steps, from obtaining food, to planning and preparing meals, to finally serving and eating the meal ${ }^{(1)}$. Food prepared at home is generally healthier than food obtained away from home ${ }^{(2-5)}$, suggesting that people who prepare food at home likely eat a healthier diet compared with people who obtain the majority of their meals away from home. Consequently, a myriad of obesity prevention strategies focus on avoiding fast and processed foods, as well as ensuring access to fresh produce for all Americans ${ }^{(6,7)}$. These strategies hinge on the assumption that if given the knowledge and opportunity, most people would purchase fresh ingredients and prepare food at home. Obesity prevention interventions are increasingly moving towards addressing the intermediate steps between food access and healthy eating planning meals, procuring ingredients and preparing food at home ${ }^{(8,9)}$. Little research, however, has examined current patterns of home food preparation in the USA and the factors affecting this complex behaviour; such work is necessary to ensure that interventions can be appropriately tailored to the needs of various groups. 
Time spent on cooking in the USA declined by almost $40 \%$ from 1965 to $1995^{(10)}$. While time-saving devices such as microwave ovens and food processors have allowed for greater efficiency, decreased frequency of cooking is likely the driving force behind this sharp decline. With ever busier lives, employed mothers' likelihood of allotting time for food preparation depends on other competing priorities, as well as their self-efficacy related to cooking and nutrition ${ }^{(11)}$. Parents may use food choice as a way to cope with stressors at home and work, using fast food or TV dinners as a reward for a difficult day or week ${ }^{(12)}$. Cooking has declined despite evidence that regular family meals lead to healthier diets and lower BMI in children and adolescents ${ }^{(13-17)}$, although most studies about family meals do not delineate whether mealtime food is prepared at home or obtained outside the home. Efforts to elucidate the relationship between home food preparation and BMI have had mixed results. A study among African American adolescents from low-income families in Baltimore showed that an increased frequency of home food preparation alone was associated with a higher BMI, while adolescents from families who prepared food at home and used healthy cooking methods had a lower $\mathrm{BMI}^{(18)}$. Another study examining the relationship between adult Americans' time-use choices and BMI found that increasing time spent on home food preparation was associated with lower BMI for adult women only ${ }^{(19)}$. From a life course standpoint, a study in Minnesota showed that food preparation behaviours track over time, and individuals who were involved in home food preparation during emerging adulthood had healthier diets in their mid to late twenties ${ }^{(20)}$.

This body of work suggests three things. First, home food preparation patterns have changed over the past several decades: time spent on cooking has declined ${ }^{(21)}$, and cooking skills and knowledge have eroded ${ }^{(11)}$. Second, home food preparation may be an important predictor of eating a healthful diet ${ }^{(2-5)}$. Third, the relationship between BMI and home food preparation is complex, variable and requires further study ${ }^{(18,19)}$. In addition, the literature reveals a broad assumption that marginalized populations, including minority and low socio-economic status (SES) groups, are more likely to engage in obesogenic behaviours and, conversely, higher-SES groups are more likely to engage in healthy behaviours. While this assumption has proven true for many health-related behaviours, including fast-food consumption $^{(22)}$ and physical activity ${ }^{(22,23)}$, variability in home food preparation across subsets of the population has not been examined.

Understanding patterns of health-related behaviours on a population level is crucial for designing, and appropriately targeting, public health interventions and policy. We therefore employed a large, nationally representative data source to examine the relationship between home food preparation and SES, race/ethnicity, family structure and other sociodemographic factors.

\section{Methods}

\section{Study population}

We analysed the prevalence and patterns of cooking dinner at home in the USA. We used National Health and Nutrition Examination Survey (NHANES) data pooled from 2007 and 2008, the only years of available data during which the outcome of interest was measured. NHANES is a nationally representative, cross-sectional data set collected by the National Center for Health Statistics. NHANES began in the 1970s and has been administered annually since 1999. Complex, multistage probability sampling is used to enrol approximately 5000 people from fifteen counties in the USA each year ${ }^{(24)}$. Specific individuals and counties vary from year to year. All ages are included. The sample is representative of the US civilian, non-institutionalized population. An average of 1.6 individuals are included from each household. In 2007 and 2008, 12943 individuals were selected and 10149 of them were interviewed. Our sample includes the households of all 10149 interviewed participants.

\section{Outcome}

The primary outcome was self-reported frequency of cooking dinner at home. This was determined during an in-person, household interview, by asking, 'During the past seven days, how many times did you or someone else in your family cook food for dinner or supper at home? This includes time putting ingredients together to cook a meal. Do not include heating up leftovers' ${ }^{, 25)}$. One reference person from each household answered this and other household-level questions. It is unknown whether the household reference person was the main food preparer in the household; for the purposes of the present analysis, we have assumed that responses will not be significantly affected by the cooking status of the household reference person. Possible answers ranged from 0 to 7 dinners cooked at home per week; $98.5 \%$ of participants responded.

\section{Covariates}

We included sociodemographic covariates describing the household whenever possible because the outcome, cooking dinner at home, is a household activity. SES was measured using the household poverty level and the household reference person's education level. The household reference person's employment status was not available for inclusion. We categorized poverty level into three groups: below 130\% of the US Census Bureau federal poverty level (FPL), 130-350\% of FPL and above $350 \%$ of FPL. Households below $130 \%$ of FPL are eligible for Supplemental Nutrition Assistance Program benefits. The household reference person's education level was categorized as: less than 9th grade, 9th-11th grade, high-school graduate or general equivalency degree (GED), some college or Associate's degree, and college graduate or above. 
The household reference person was always aged 18 years or older. We categorized age into four groups: 18-25 years, 26-45 years, 46-65 years and over 65 years. We included race/ethnicity and country of birth as proxies of culture. The household reference person's race/ethnicity was not available for inclusion, so the study participant's race/ethnicity was used. Race/ethnicity was self-reported as white, black, Hispanic or other. The household reference person's country of birth was reported as born in the USA, born in a Spanish-speaking country, or born in a foreign, non-Spanish-speaking country. We collapsed country of birth into two categories: US-born or foreign-born.

We included marital status and the presence of dependants in the household as indicators of family structure. We categorized the household reference person's marital status into two groups: either living with a partner (living with a partner or married) or living without a partner (widowed, divorced, separated or never married). We inferred the presence of dependants in the household by subtracting 1 from the household size if the reference person was not living with a partner and by subtracting 2 if the reference person was living with a partner. If the resulting number was greater than or equal to 1 , we classified that household as having dependants.

We also included household food security, which was determined using the US Food Security Survey module and was categorized as very low, low, marginal or full food security.

\section{Statistical analysis}

All analyses accounted for the complex NHANES survey design, including stratification, cluster design and weighting, producing estimates and standard errors representative of the non-institutionalized US population ${ }^{(24)}$. We examined frequency of cooking dinner at home as an ordinal, count variable, ranging from 0 to 7 dinners cooked at home per week. We calculated the prevalence of each frequency of cooking dinner at home, as well as the mean number of dinners cooked per week by various sociodemographic groups, using the covariates above.

We then collapsed the frequency of cooking dinner at home into three categories, comparing households that never cook dinner $(0-1$ dinners cooked per week) or always cook dinner (6-7 dinners cooked per week), with households that sometimes cook dinner at home (2-5 dinners cooked per week). We determined the distribution of each sociodemographic group across dinner categories and fit a multinomial logistic regression model to test associations between cooking dinner at home and the sociodemographic covariates. We tested for interactions between SES variables and race/ethnicity. We performed fractional polynomial regression to better understand the relationship between SES and cooking dinner at home, by plotting the predicted mean household poverty level and education level based on the frequency of home dinner preparation. All analyses were done using the statistical software package Stata $11 \cdot 2$.

\section{Results}

On a population level, $8 \%$ of Americans reported never cooking dinner, $43 \%$ sometimes cooking dinner and 49\% always cooking dinner at home (Tables 1 and 2). There were differences in the average number of dinners cooked at home per week across many of the covariates (Table 3). Respondents from households below 130\% of FPL cooked an average of 5.4 dinners per week (95\% CI $5 \cdot 2,5 \cdot 7)$ compared with $4 \cdot 8$ dinners per week for households over $350 \%$ of FPL (95\% CI $4 \cdot 6,5 \cdot 0$ ). Respondents from households where the reference person had less than a 9th grade education cooked an average of $6 \cdot 0$ dinners per week (95\% CI $5 \cdot 7,6 \cdot 2)$, compared with $4 \cdot 9$ dinners per week $(95 \% \mathrm{CI} 4 \cdot 7,5 \cdot 1)$ if the reference person attended at least some college. Respondents from black households cooked the fewest dinners at home (mean $4 \cdot 4$, 95\% CI 4.2, 4.6). Respondents from Hispanic households with a foreign-born reference person cooked more (mean $5 \cdot 9,95 \%$ CI $5 \cdot 8,6 \cdot 1$ ) than respondents from Hispanic households with a US-born reference person (mean $4 \cdot 9$, $95 \%$ CI $4 \cdot 7,5 \cdot 1$ ), who cooked at a rate similar to whites (mean $5 \cdot 0,95 \% \mathrm{CI} 4 \cdot 8,5 \cdot 2$ ). In general, respondents from households where the reference person was US-born cooked an average of 4.9 dinners per week (95\% CI $4 \cdot 7$, $5 \cdot 1$ ), compared with $5 \cdot 8$ dinners per week (95\% CI $5 \cdot 7$, $6 \cdot 0)$ if the reference person was foreign-born. Respondents from households with dependants cooked more often (mean 5.2, 95\% CI 5.1, 5.4) than respondents who lived alone and respondents from households without dependants (mean 4·6, $95 \%$ CI 4·3, 5•0).

Frequency of home dinner preparation varied across the SES spectrum (Figs 1 and 2). Poorer, less educated households were more likely to either always or never

Table 1 Prevalence of home dinner preparation in the USA; National Health and Nutrition Examination Survey (NHANES) $2007-2008^{*}+$

\begin{tabular}{lcc}
\hline $\begin{array}{l}\text { Frequency of cooking dinner } \\
\text { at home each week }\end{array}$ & \% of US population \\
\hline Never cook & 5 & 8 \\
$\quad 0$ & 3 & \\
1 & 5 & \\
Sometimes cook & 8 & \\
2 & 13 & \\
3 & 17 & \\
4 & 13 & \\
5 & 36 & \\
Always cook & & \\
6 & & \\
7 & & \\
& ${ }^{*} n$ 9993, population size $=293699$ 077. \\
†All analyses were completed with appropriate weighting, taking into \\
account the complex survey design of NHANES 2007-2008.
\end{tabular}


Table 2 Sociodemographic characteristics of the total sample and in each dinner preparation category*; National Health and Nutrition Examination Survey (NHANES) 2007-2008

\begin{tabular}{|c|c|c|c|c|}
\hline & \multirow[b]{2}{*}{ Total sample } & \multicolumn{3}{|c|}{ Frequency of cooking dinner at home each week } \\
\hline & & \multicolumn{3}{|c|}{$\%$ of US population } \\
\hline & $\%$ of US population & Never cook $(0-1)$ & Sometimes cook (2-5) & Always cook $(6-7)$ \\
\hline Total (\%) & 100 & $8 \cdot 0$ & 43 & 49 \\
\hline \multicolumn{5}{|l|}{ Federal poverty levelt (\%) } \\
\hline$<130 \%$ & 24 & 30 & 15 & 32 \\
\hline $130-350 \%$ & 35 & 38 & 35 & 35 \\
\hline$>350 \%$ & 41 & 32 & 51 & 33 \\
\hline \multicolumn{5}{|l|}{ Educationt (\%) } \\
\hline$<9$ th grade & $7 \cdot 1$ & $5 \cdot 8$ & $2 \cdot 8$ & 11 \\
\hline 9 th -11 th grade & 13 & 16 & 10 & 16 \\
\hline High-school graduate or GED & 26 & 29 & 24 & 27 \\
\hline Some college or Associate's degree & 28 & 30 & 32 & 25 \\
\hline College graduate or above & 25 & 18 & 31 & 22 \\
\hline \multicolumn{5}{|l|}{ Gendert (\%) } \\
\hline Male & 45 & 48 & 43 & 47 \\
\hline Female & 55 & 52 & 57 & 53 \\
\hline \multicolumn{5}{|l|}{ Aget $(\%)$} \\
\hline $18-25$ years & $7 \cdot 5$ & 18 & $7 \cdot 8$ & $5 \cdot 6$ \\
\hline $26-45$ years & 45 & 36 & 47 & 45 \\
\hline $46-65$ years & 34 & 28 & 36 & 33 \\
\hline$>65$ years & 13 & 18 & $9 \cdot 0$ & 16 \\
\hline \multicolumn{5}{|l|}{ Race/ethnicity+ (\%) } \\
\hline White & 66 & 59 & 72 & 63 \\
\hline Black & 12 & 23 & 14 & $9 \cdot 4$ \\
\hline Hispanic & 15 & 15 & 10 & 20 \\
\hline Other & $6 \cdot 2$ & 3.2 & $5 \cdot 0$ & $7 \cdot 8$ \\
\hline \multicolumn{5}{|l|}{ Country of birtht (\%) } \\
\hline USA & 18 & 14 & $9 \cdot 9$ & 25 \\
\hline Foreign born & 82 & 86 & 90 & 75 \\
\hline \multicolumn{5}{|l|}{ Living with a partnert (\%) } \\
\hline No & 30 & 58 & 30 & 26 \\
\hline Yes & 70 & 42 & 70 & 74 \\
\hline \multicolumn{5}{|l|}{ Living with dependantst (\%) } \\
\hline No & 29 & 49 & 29 & 25 \\
\hline Yes & 71 & 51 & 71 & 75 \\
\hline \multicolumn{5}{|l|}{ Household food security† (\%) } \\
\hline Very low & $4 \cdot 2$ & $5 \cdot 9$ & $3 \cdot 6$ & 4.5 \\
\hline Low & $9 \cdot 3$ & $5 \cdot 2$ & $7 \cdot 7$ & 11 \\
\hline Marginal & $8 \cdot 0$ & $7 \cdot 4$ & $5 \cdot 5$ & 10 \\
\hline Full & 79 & 81 & 83 & 74 \\
\hline
\end{tabular}

GED, general equivalency degree.

*All analyses were completed with appropriate weighting, taking into account the complex survey design of NHANES 2007-2008.

tDifferences in the percentage of the sociodemographic group present in each dinner category were significant at $P<0 \cdot 001$ by the Wald $\chi^{2}$ test.

cook dinner at home, whereas wealthier, more educated households were more likely to sometimes cook dinner at home. This pattern was evident across all self-reported race and ethnicity groups.

In the multinomial regression, we determined the likelihood of always or never cooking dinner at home, compared with sometimes cooking dinner at home (Table 4). Adjusting for the other covariates, several characteristics were significantly associated with a greater likelihood of always cooking dinner at home: income less than $130 \%$ of FPL (relative risk $(\mathrm{RR})=3 \cdot 03,95 \%$ CI $2 \cdot 13$, $4 \cdot 31, P<0 \cdot 0001)$, income $130-350 \%$ of FPL $(\mathrm{RR}=1 \cdot 39$, $95 \%$ CI $0.99,1 \cdot 93, P=0 \cdot 05), 11$ th grade education or less ( $<9$ th grade: $\mathrm{RR}=1 \cdot 77,95 \%$ CI $1 \cdot 05,2 \cdot 99, P=0 \cdot 03$; 9th-11th grade: $\mathrm{RR}=1 \cdot 54,95 \% \mathrm{CI} 1 \cdot 12,2 \cdot 14, P=0 \cdot 01$ ), age greater than 45 years (age $45-65$ years: $R R=1 \cdot 32$, $95 \%$ CI $1 \cdot 12,1 \cdot 54, P=0 \cdot 002$; age $>65$ years: $\mathrm{RR}=2 \cdot 51$,
95\% CI $2 \cdot 14,2 \cdot 95, P<0 \cdot 0001$ ), having a foreign-born household reference person ( $\mathrm{RR}=2 \cdot 42,95 \%$ CI $1 \cdot 68$, $3.51, P<0.0001)$, living with a partner $(\mathrm{RR}=1.63,95 \%$ CI $1 \cdot 27,2 \cdot 10, P=0 \cdot 001)$ and having marginal food security (RR $=1 \cdot 43,95 \% \mathrm{CI} 1 \cdot 01,2 \cdot 01, P=0 \cdot 04)$. On the other hand, income greater than $350 \%$ of FPL, male gender and black race were all associated with a lower likelihood of always cooking dinner (male: $\mathrm{RR}=0.81$, $95 \%$ CI $0 \cdot 70,0 \cdot 94, P=0 \cdot 01$; black: $\mathrm{RR}=0 \cdot 62,95 \% \mathrm{CI}$ $0.53,0.73, P<0 \cdot 0001)$.

Characteristics significantly associated with a greater likelihood of never cooking dinner at home were: income less than $130 \%$ of FPL $(\mathrm{RR}=1 \cdot 80,95 \% \mathrm{CI} 1 \cdot 18,2 \cdot 76$, $P=0 \cdot 01$ ), 9th-12th grade education (9th-11th grade: $\mathrm{RR}=1 \cdot 83,95 \%$ CI $1 \cdot 11,3 \cdot 04, \quad P=0 \cdot 02$; high-school graduate or GED: $\mathrm{RR}=1 \cdot 84,95 \% \mathrm{CI} 1 \cdot 03,3 \cdot 29, P=0 \cdot 04$ ), age $18-25$ years $(\mathrm{RR}=1 \cdot 86,95 \% \mathrm{CI} 1 \cdot 07,3 \cdot 23, P=0 \cdot 03)$, 
Table 3 Frequency of home dinner preparation by sociodemographic group*; National Health and Nutrition Examination Survey (NHANES) 2007-2008

\begin{tabular}{|c|c|c|c|c|c|c|}
\hline & \multirow[b]{3}{*}{$n$} & & & \multicolumn{3}{|c|}{ Frequency of cooking dinner at home each weekt } \\
\hline & & \multicolumn{2}{|c|}{ Dinners cooked per weekł } & \multicolumn{3}{|c|}{$\%$ of US population } \\
\hline & & Mean & $95 \% \mathrm{Cl}$ & Never cook $(0-1)$ & Sometimes cook $(2-5)$ & Always cook (6-7) \\
\hline Total & 10149 & $5 \cdot 0$ & $4 \cdot 9,5 \cdot 2$ & $7 \cdot 8$ & 43 & 49 \\
\hline \multicolumn{7}{|l|}{ Federal poverty level§̧ } \\
\hline$<130 \%$ & 3449 & $5 \cdot 4$ & $5 \cdot 2,5 \cdot 7$ & $9 \cdot 4$ & 26 & 64 \\
\hline $130-350 \%$ & 3440 & $5 \cdot 0$ & $4 \cdot 8,5 \cdot 3$ & $8 \cdot 5$ & 44 & 48 \\
\hline$>350 \%$ & 2366 & $4 \cdot 8$ & $4 \cdot 6,5 \cdot 0$ & $6 \cdot 1$ & 54 & 39 \\
\hline \multicolumn{7}{|l|}{ Education§ } \\
\hline$<9$ th grade & 1205 & $6 \cdot 0$ & $5 \cdot 7,6 \cdot 2$ & $6 \cdot 3$ & 17 & 76 \\
\hline 9th-11th grade & 1778 & $5 \cdot 3$ & $5 \cdot 0,5 \cdot 6$ & $9 \cdot 2$ & 33 & 58 \\
\hline High-school graduate or GED & 2459 & $5 \cdot 0$ & $4 \cdot 8,5 \cdot 2$ & $8 \cdot 8$ & 40 & 51 \\
\hline Some college or Associate's degree & 2539 & 4.9 & $4 \cdot 6,5 \cdot 1$ & $8 \cdot 2$ & 49 & 43 \\
\hline College graduate or above & 1751 & 4.9 & $4 \cdot 7,5 \cdot 1$ & $5 \cdot 5$ & 52 & 43 \\
\hline \multicolumn{7}{|l|}{ Gender§ } \\
\hline Male & 5157 & $5 \cdot 1$ & $4 \cdot 9,5 \cdot 3$ & $8 \cdot 2$ & 40 & 51 \\
\hline Female & 4992 & $5 \cdot 0$ & $4 \cdot 9,5 \cdot 1$ & $7 \cdot 3$ & 45 & 48 \\
\hline \multicolumn{7}{|l|}{ Age§ } \\
\hline $18-25$ years & 850 & $4 \cdot 2$ & $3 \cdot 8,4 \cdot 7$ & 18 & 45 & 37 \\
\hline $26-45$ years & 4835 & $5 \cdot 1$ & $5 \cdot 0,5 \cdot 2$ & $6 \cdot 1$ & 45 & 49 \\
\hline $46-65$ years & 2908 & $5 \cdot 1$ & $4 \cdot 9,5 \cdot 2$ & $6 \cdot 3$ & 45 & 48 \\
\hline$>65$ years & 1556 & $5 \cdot 2$ & $4 \cdot 9,5 \cdot 6$ & 11 & 29 & 60 \\
\hline \multicolumn{7}{|l|}{ Race/ethnicity§ } \\
\hline White & 4115 & $5 \cdot 0$ & $4 \cdot 8,5 \cdot 2$ & $6 \cdot 9$ & 46 & 47 \\
\hline Black & 2211 & $4 \cdot 4$ & $4 \cdot 2,4 \cdot 6$ & 14 & 47 & 38 \\
\hline Hispanic & 3358 & $5 \cdot 6$ & $5 \cdot 4,5 \cdot 7$ & $7 \cdot 7$ & 28 & 65 \\
\hline Other & 465 & $5 \cdot 6$ & $5 \cdot 4,6 \cdot 0$ & $4 \cdot 0$ & 34 & 62 \\
\hline \multicolumn{7}{|l|}{ Country of birth§ } \\
\hline USA & 7109 & $4 \cdot 9$ & $4 \cdot 7,5 \cdot 1$ & $8 \cdot 0$ & 47 & 45 \\
\hline Foreign born & 2637 & $5 \cdot 8$ & $5 \cdot 7,6 \cdot 0$ & $5 \cdot 8$ & 24 & 70 \\
\hline \multicolumn{7}{|l|}{ Living with a partner§ } \\
\hline No & 3260 & $4 \cdot 5$ & $4 \cdot 2,4 \cdot 8$ & 14 & 43 & 43 \\
\hline Yes & 6362 & $5 \cdot 3$ & $5 \cdot 2,5 \cdot 4$ & $4 \cdot 5$ & 43 & 53 \\
\hline \multicolumn{7}{|l|}{ Living with dependants $\$$} \\
\hline No & 2211 & $4 \cdot 6$ & $4 \cdot 3,5 \cdot 0$ & 13 & 44 & 44 \\
\hline Yes & 7411 & $5 \cdot 2$ & $5 \cdot 1,5 \cdot 4$ & $5 \cdot 3$ & 43 & 52 \\
\hline \multicolumn{7}{|l|}{ Household food security§ } \\
\hline Very low & 627 & $5 \cdot 0$ & $4 \cdot 5,5 \cdot 5$ & 11 & 37 & 52 \\
\hline Low & 1339 & $5 \cdot 4$ & $5 \cdot 0,5 \cdot 8$ & $4 \cdot 3$ & 36 & 60 \\
\hline Marginal & 1141 & $5 \cdot 5$ & $5 \cdot 2,5 \cdot 8$ & $7 \cdot 2$ & 30 & 63 \\
\hline Full & 6882 & $5 \cdot 0$ & $4 \cdot 8,5 \cdot 1$ & $8 \cdot 1$ & 46 & 46 \\
\hline
\end{tabular}

GED, general equivalency degree.

${ }^{*}$ All analyses were completed with appropriate weighting, taking into account the complex survey design of NHANES 2007-2008.

tWe calculated the percentage of each sociodemographic group in each dinner category.

$\ddagger$ We calculated the mean number of dinners cooked per week and the $95 \%$ confidence interval for each sociodemographic group.

$\S$ Differences in the percentage of the sociodemographic group present in each dinner category were significant at $P<0 \cdot 001$ by the Wald $\chi^{2}$ test.

black race $(\mathrm{RR}=1 \cdot 75,95 \% \mathrm{CI} 1 \cdot 14,2 \cdot 68, P=0 \cdot 01)$ and Hispanic ethnicity $(\mathrm{RR}=1 \cdot 89,95 \%$ CI $1 \cdot 01,3 \cdot 53, P=0 \cdot 05)$. On the other hand, several characteristics were associated with being less likely to never cook dinner at home: if the reference person lived with a partner $(\mathrm{RR}=0 \cdot 42,95 \% \mathrm{CI}$ $0 \cdot 28,0 \cdot 64, P<0 \cdot 0001)$, if there were dependants in the household ( $\mathrm{RR}=0 \cdot 36,95 \% \mathrm{CI} 0 \cdot 26,0 \cdot 50, P<0 \cdot 0001)$ and if there was low food security $(\mathrm{RR}=0 \cdot 49,95 \%$ CI $0 \cdot 27$, $0 \cdot 88, P=0 \cdot 02)$. Interaction terms between SES variables and race were not significant (data not shown).

\section{Discussion}

Based on this nationally representative sample from 2007 and 2008, patterns of home dinner preparation in the USA varied substantially with SES. Poorer, less educated households were more likely to either always or never cook dinner at home, and wealthier, more educated households were more likely to sometimes cook dinner at home. On the whole, half of American households always cooked dinner at home. Dinner preparation patterns also varied with family structure and culture. Households with dependants were much less likely to never cook dinner at home, and households where the reference person lived with a partner were more likely to always cook dinner at home. Being born outside the USA was associated with cooking more dinners at home. Interestingly, respondents from Hispanic households with a foreign-born reference person cooked the most dinners at home, while respondents from Hispanic households with a US-born reference person cooked 

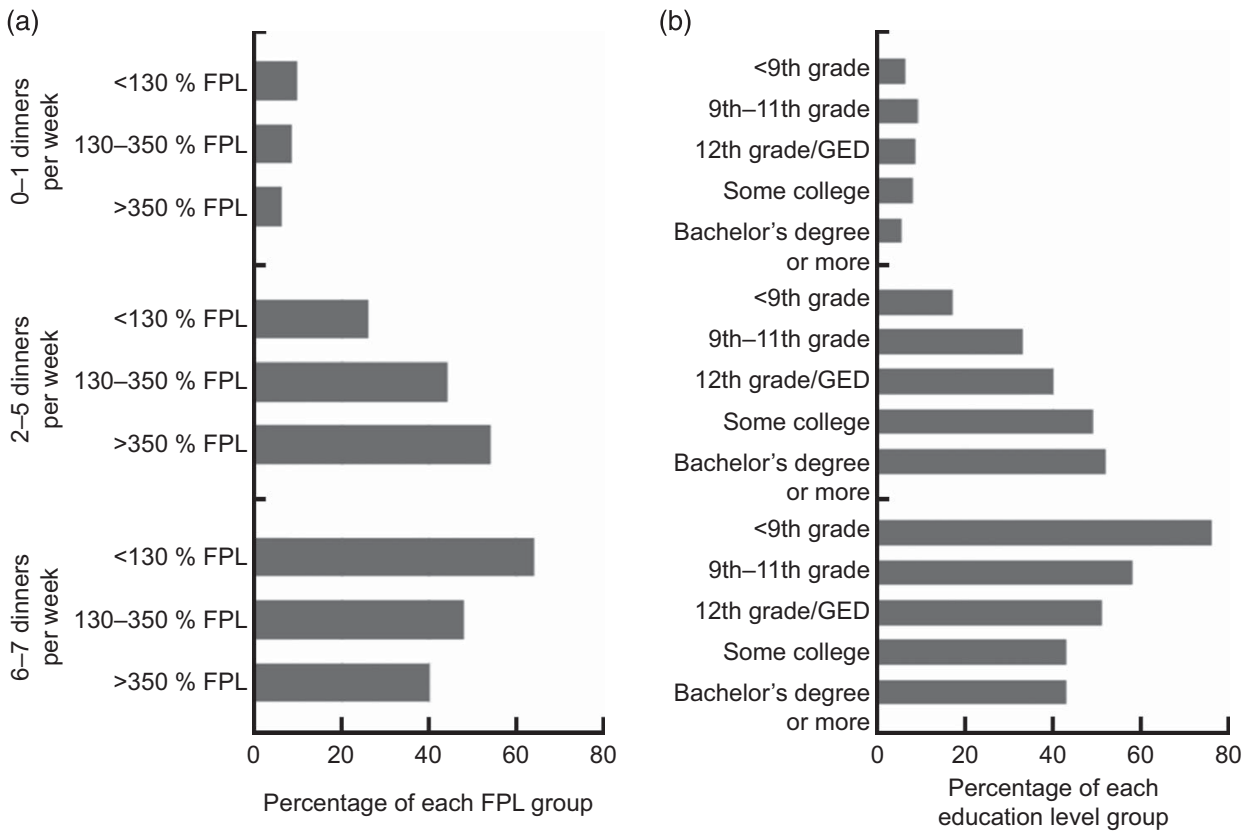

Fig. 1 Socio-economic status (SES) and home dinner preparation in the USA; National Health and Nutrition Examination Survey (NHANES) 2007-2008. We plotted the percentage of each SES group, stratified by (a) federal poverty level (FPL) and (b) education level, in each dinner category $(0-1,2-5$ or 6-7 dinners cooked at home per week). The corresponding numbers are found in Table 3; all differences were significant at $P<0.001$ by the Wald $\chi^{2}$ test (GED, general equivalency degree)

(a)
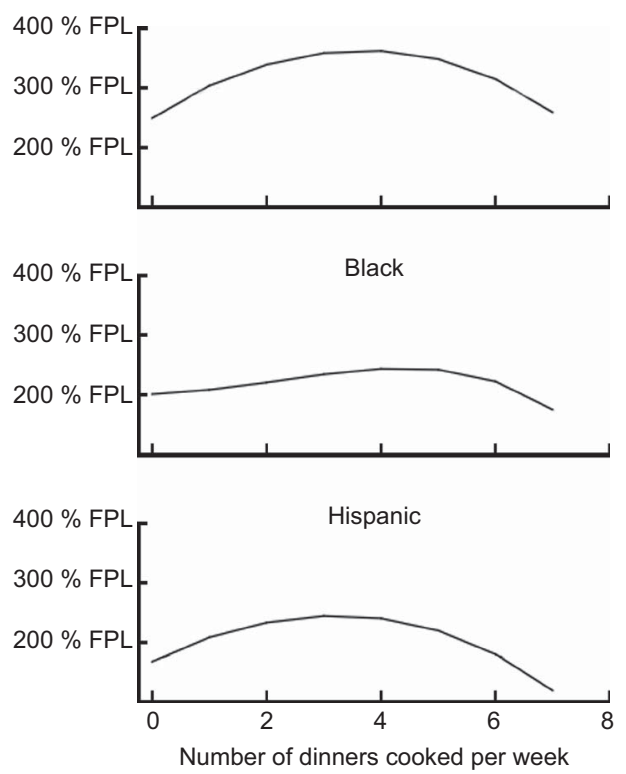

(b)
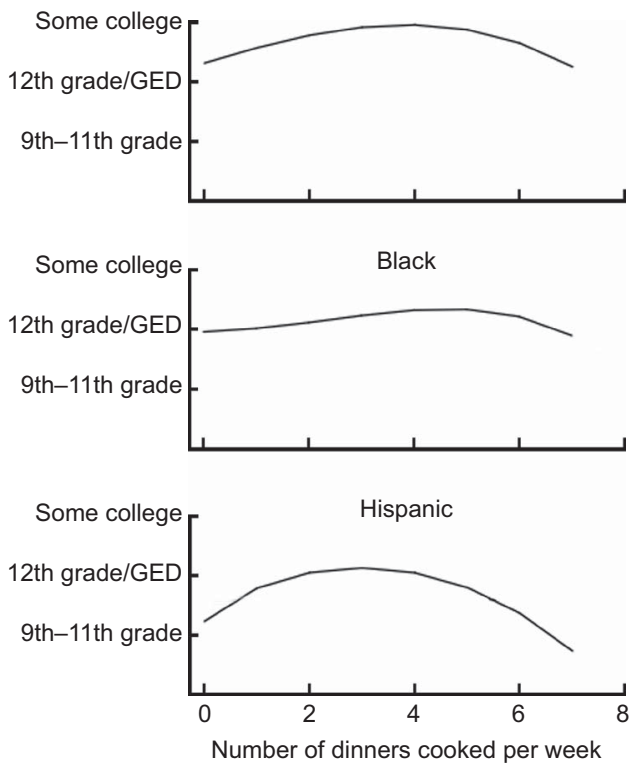

Fig. 2 Predicted socio-economic status based on frequency of home dinner preparation in the USA; National Health and Nutrition Examination Survey (NHANES) 2007-2008. We stratifed by race/ethnicity and used fractional polynomial regression to predict (a) the mean expected household federal poverty level (FPL) and (b) the mean expected education level based on the number of dinners cooked at home per week (GED, general equivalency degree)

at a rate similar to whites. Respondents from black households cooked the least.

The relative preponderance of cooking, a healthy behaviour $^{(2-4)}$, among low-SES households runs counter to the widely held assumption that low-SES groups are more likely to engage in unhealthy behaviours and high-SES groups are more likely to engage in healthy behaviours ${ }^{(22,23)}$. Confounding is unlikely to account for these observed relationships, which in our study hold true even when controlling for factors such as race/ethnicity 
Table 4 Associations between sociodemographic characteristics and frequency of home dinner preparation*; National Health and Nutrition Examination Survey (NHANES) 2007-2008

\begin{tabular}{|c|c|c|c|c|c|c|c|}
\hline & \multicolumn{7}{|c|}{ Frequency of cooking dinner at home each week } \\
\hline & \multicolumn{3}{|c|}{ Never cook $(0-1)$} & \multirow{2}{*}{$\frac{\text { Sometimes cook }(2-5)}{\text { Reference }}$} & \multicolumn{3}{|c|}{ Always cook (6-7) } \\
\hline & $\mathrm{RR}$ & $95 \% \mathrm{Cl}$ & $P$ value & & RR & $95 \% \mathrm{Cl}$ & $P$ value \\
\hline \multicolumn{8}{|l|}{ Federal poverty level } \\
\hline$<130 \%$ & $1 \cdot 80$ & $1 \cdot 18,2 \cdot 76$ & 0.01 & $1 \cdot 00$ & $3 \cdot 03$ & $2 \cdot 13,4 \cdot 31$ & $<0 \cdot 0001$ \\
\hline $130-350 \%$ & $1 \cdot 13$ & $0 \cdot 74,1 \cdot 74$ & 0.54 & $1 \cdot 00$ & $1 \cdot 39$ & $0.99,1.93$ & 0.05 \\
\hline & \multicolumn{7}{|c|}{ Education } \\
\hline 9 th -11 th grade & 1.83 & $\begin{array}{l}0.03,3.03 \\
1.11,3.04\end{array}$ & $\begin{array}{l}0.14 \\
0.02\end{array}$ & 1.00 & 1.54 & $\begin{array}{l}1.05,2 \cdot 99 \\
1 \cdot 12 \\
1 \cdot 14\end{array}$ & 0.03 \\
\hline High-school graduate or GED & $1 \cdot 84$ & $1.03,3.29$ & 0.04 & 1.00 & 1.22 & $0.75,2 \cdot 00$ & 0.40 \\
\hline Some college or Associate's degree & $1 \cdot 34$ & $0 \cdot 82,2 \cdot 19$ & 0.23 & $1 \cdot 00$ & 0.97 & $0 \cdot 68,1 \cdot 27$ & 0.85 \\
\hline College graduate or above & Reference & & & & Reference & & \\
\hline \multicolumn{8}{|l|}{ Gender } \\
\hline Male & 1.09 & $0 \cdot 73,1 \cdot 64$ & $0 \cdot 65$ & $1 \cdot 00$ & $0 \cdot 81$ & $0 \cdot 70,0.94$ & 0.01 \\
\hline Female & Reference & & & & Reference & & \\
\hline \multicolumn{8}{|l|}{ Age } \\
\hline $\begin{array}{l}18-25 \text { years } \\
26-45 \text { years }\end{array}$ & $\begin{array}{c}1 \cdot 86 \\
\text { Reference }\end{array}$ & $1 \cdot 07,3 \cdot 23$ & 0.03 & $1 \cdot 00$ & $\begin{array}{c}0.75 \\
\text { Reference }\end{array}$ & $0 \cdot 46,1 \cdot 21$ & $0 \cdot 22$ \\
\hline $46-65$ years & 0.82 & $0.50,1.34$ & 0.41 & $1 \cdot 00$ & $1 \cdot 32$ & $1 \cdot 12,1 \cdot 54$ & 0.002 \\
\hline$>65$ years & $1 \cdot 39$ & $0 \cdot 75,2 \cdot 56$ & 0.27 & 1.00 & $2 \cdot 51$ & $2 \cdot 14,2 \cdot 95$ & $<0.0001$ \\
\hline \multicolumn{8}{|l|}{ Race/ethnicity } \\
\hline White & Reference & & & & Reference & & \\
\hline Black & $1 \cdot 75$ & $1 \cdot 14,2 \cdot 68$ & 0.01 & $1 \cdot 00$ & 0.62 & $0.53,0.73$ & $<0.0001$ \\
\hline Hispanic & $1 \cdot 89$ & $1 \cdot 01,3.53$ & 0.05 & $1 \cdot 00$ & 0.95 & $0 \cdot 67,1 \cdot 33$ & $0 \cdot 74$ \\
\hline Other & $0 \cdot 78$ & $0 \cdot 35,1 \cdot 74$ & 0.52 & 1.00 & 1.05 & $0.68,1 \cdot 62$ & $0 \cdot 81$ \\
\hline \multicolumn{8}{|l|}{ Country of birth } \\
\hline USA & Reference & & & & Reference & & \\
\hline Foreign born & $1 \cdot 56$ & $0 \cdot 81,3 \cdot 01$ & $0 \cdot 17$ & $1 \cdot 00$ & $2 \cdot 42$ & $1 \cdot 68,3 \cdot 51$ & $<0.0001$ \\
\hline \multicolumn{8}{|l|}{ Living with a partner } \\
\hline No & Reference & & & & Reference & & \\
\hline Yes & 0.42 & $0.28,0.64$ & $<0.0001$ & $1 \cdot 00$ & $1 \cdot 63$ & $1 \cdot 27,2 \cdot 10$ & $0 \cdot 001$ \\
\hline \multicolumn{8}{|l|}{ Living with dependants } \\
\hline No & Reference & & & & Reference & & \\
\hline Yes & 0.36 & $0.26,0.50$ & $<0.0001$ & 1.00 & $1 \cdot 27$ & $0.95,1.72$ & $0 \cdot 10$ \\
\hline \multicolumn{8}{|l|}{ Household food security } \\
\hline Very low & 0.93 & $0.61,1 \cdot 42$ & $0 \cdot 71$ & $1 \cdot 00$ & 0.83 & $0 \cdot 50,1 \cdot 39$ & 0.46 \\
\hline Low & 0.49 & $0 \cdot 27,0 \cdot 88$ & 0.02 & 1.00 & $1 \cdot 01$ & $0.66,1.55$ & 0.95 \\
\hline Marginal & 0.94 & $0.45,1.97$ & $0 \cdot 86$ & 1.00 & $1 \cdot 43$ & $1 \cdot 01,2 \cdot 01$ & 0.04 \\
\hline Full & Reference & & & & Reference & & \\
\hline
\end{tabular}

RR, relative risk; GED, general equivalency degree.

${ }^{*}$ Relative risks and $95 \%$ confidence intervals were calculated using multinomial regression models, with households that cook $2-5$ dinners at home per week as the reference group.

and family structure. Previous studies have shown that time is a significant barrier preventing many people from cooking, and this is especially true among low-SES groups ${ }^{(10-12)}$. Our work further explains these observations by showing that compared with high-SES groups, low-SES groups have a more dichotomous (never/always) distribution when it comes to cooking. One possible explanation for this finding is the differential value of time and money across SES strata, whereby for low-SES groups, the marginal value of time may be lower than the marginal value of money. That is, although cooking at home consumes more time, money is scarcer than time. There are only two ways to maximize food dollars in this setting: (i) cook at home or (ii) obtain cheap (often less healthy) food outside the home. Cooking allows limited food dollars to be stretched further. The opposite would be true in high-SES groups. With the emergence of discretionary income, the marginal value of time is greater than the marginal value of money, leading high-SES groups to be more likely to sometimes cook dinner at home and sometimes not. Assuming an equal desire to eat healthily and an equal ability to prepare food at home, variation in the marginal value of time and money may explain some of the variation in home food preparation patterns by SES.

Cooking at home can therefore represent either a choice or a forced option. Households with increased means via greater wealth and education choose to eat something other than a home-cooked meal several nights per week, while poorer, less educated households are forced to either always or never cook dinner at home. We found that while half of all Americans always cook dinner at home, the remainder obtain many or most dinners away from home. The well-described increase in processed and fast-food consumption over the past 
several decades suggests a concomitant decline in preparing food at home ${ }^{(26)}$, and our data fit that pattern. On one hand, it is encouraging that despite the constraints of modern society, many Americans still find it possible to regularly prepare dinner at home. Yet the choice of wealthier members of US society not to cook at home is a choice to move towards something, be it leisure, less housework or more free time. Any effective public policy response to motivate home cooking as a means to healthy eating will have to account for these incentives.

Never or always cooking dinner at home also appears to be a forced option for populations marginalized by race or ethnicity. We found that households self-reporting as black or Hispanic were more likely to never cook dinner at home. The effect of Hispanic ethnicity was observed only after adjusting for being foreign-born, which is not surprising given that Hispanic households with a foreign-born reference person cooked much more than Hispanic households with a US-born reference person. This suggests that acculturation into a marginalized segment of American society may decrease the frequency of cooking dinner at home. Variation by race and country of birth supports the idea that cultural norms have a significant effect on people's cooking patterns, regardless of other factors ${ }^{(27,28)}$.

Family structure also plays a significant role in home food preparation habits. Households with dependants were one-third as likely to never cook dinner at home, and households where the reference person lived with a partner were 1.6 times more likely to always cook dinner at home. These data are consistent with the idea that cooking and feeding others are inherently social behaviours driven by a desire to provide both emotional and material stability, and that those who live in a social environment will therefore be more likely to $\operatorname{cook}^{(1)}$.

The present study has both limitations and strengths. First is the certain existence of unmeasured confounders not reported in this data source, such as information about the local food environment, urban/suburban/ rural location, household time constraints, and attitudes regarding the importance of health, nutrition and home-cooked meals. Second, the outcome, frequency of home dinner preparation, was based on self-report and therefore vulnerable to recall bias, social desirability and varying interpretations of the question, in particular the definition of cooking. Third, because the data are observational and cross-sectional, we can only observe associations and cannot infer causality. These relatively recent, nationally representative data do allow, however, for a current appraisal of home food preparation that is generalizable to the non-institutionalized US population. The wide reach and rapidly evolving nature of the obesity epidemic make it important to have a current, population-level understanding of health-related behaviours. The present study's most significant contribution is providing insight into the factors affecting home food preparation, an under-examined behaviour in the research on healthy eating and obesity.

Preparing healthy food at home is potentially one of the most significant points for effective interventions to curb the obesity epidemic, both at the individual and population level. Distinct patterns of home food preparation likely emerge because the factors driving home food preparation behaviours vary across different populations. Interventions aimed at increasing home food preparation will therefore need to be tailored and targeted to meet the unique needs of specific groups. The presumption of the ability and desire to prepare food at home is implicit in many obesity prevention strategies, including the national initiative to eradicate food deserts in the USA by $2017^{(6)}$. The potential effectiveness of this initiative hinges on the assumption that if given access, families will purchase fresh ingredients and prepare healthy food at home. Recent data do not, however, show a consistent relationship between geographic proximity to fresh food and healthy eating ${ }^{(7,29-31)}$. Providing access to fresh ingredients is only the first step. Ensuring that people will choose to prepare food at home, operating within constraints of money and time, will help translate access into healthy eating. While many programmes incorporate food preparation ability into a broader agenda to improve healthy eating and active living, little research has focused directly on the home food preparation aspect of the intervention. A recent Cochrane review found that broad-based obesity prevention and intervention programmes in children have had mixed success, further underscoring the importance of systematically designing and tailoring intervention programmes to meet individual needs ${ }^{(32)}$.

There are four main considerations for future studies. First, although previous studies have shown that food prepared at home is generally healthier than food obtained away from home, we need to establish whether this relationship holds true across SES strata, cultural groups and family structures. Second, the relationship between home food preparation and health outcomes such as obesity, CVD and diabetes requires further study. Third, we must better understand the forces that shape household choices regarding home food preparation. Finally, we must design effective, enticing and scalable interventions to improve home food preparation practices across diverse groups.

\section{Acknowledgements}

Sources of funding: S.V. was supported by a National Research Service Award institutional training grant for primary medical care (T32-HP10026). No funder had a role in the design, analysis or writing of this article. Conflicts of interest: None of the authors had any conflict of interest to declare. Authors' contributions: S.V., J.A.L., D.E.P. and C.F. designed the research. S.V., M.O.H., D.E.P. 
and C.F. performed the statistical analysis. S.V., J.A.L., M.O.H., D.E.P. and C.F. wrote the manuscript. S.V. had primary responsibility for final content. All authors read and approved the final manuscript. Acknowledgements: The authors gratefully acknowledge Alexander Fiks, MD, MSCE, Lisa McLeod, MD, MSCE, and Catherine Zorc, MD, MSHP for their contributions to this manuscript.

\section{References}

1. Kaufmann J-C (2010) The Meaning of Cooking (Casseroles, amour et crises). Cambridge: Polity Press.

2. Lin B-H \& Guthrie J (1999) Quality of children's diets at and away from home: 1994-96. Food Rev 22, 2-10.

3. Bowman SA, Gortmaker SL, Ebbeling CB et al. (2004) Effects of fast-food consumption on energy intake and diet quality among children in a national household survey. Pediatrics 113, 112-118.

4. Briefel RR, Wilson A \& Gleason PM (2009) Consumption of low-nutrient, energy-dense foods and beverages at school, home, and other locations among school lunch participants and nonparticipants. J Am Diet Assoc 109, 2 Suppl., S79-S90.

5. Larson NI, Perry CL, Story M et al. (2006) Food preparation by young adults is associated with better diet quality. $J \mathrm{Am}$ Diet Assoc 106, 2001-2007.

6. Kohan EG (2011). Sam Kass: First Lady will acheive goal of eradicating all US food deserts by 2017. Obama Foodorama: The Blog Of Record About White House Food Initiatives, From Policy To Pie. http://obamafoodorama. blogspot.com/2011/10/sam-kass-first-lady-will-meet-goal-of. html (accessed November 2011).

7. Story M, Kaphingst KM, Robinson-O'Brien R et al. (2008) Creating healthy food and eating environments: policy and environmental approaches. Annu Rev Public Health 29, 253-272.

8. Fulkerson JA, Rydell S, Kubik MY et al. (2010) Healthy Home Offerings via the Mealtime Environment (HOME): feasibility, acceptability, and outcomes of a pilot study. Obesity (Silver Spring) 18, Suppl. 1, S69-S74.

9. Davis JN, Ventura EE, Cook LT et al. (2011) LA Sprouts: a gardening, nutrition, and cooking intervention for Latino youth improves diet and reduces obesity. J Am Diet Assoc 111, 1224-1230.

10. Jabs J \& Devine CM (2006) Time scarcity and food choices: an overview. Appetite 47, 196-204.

11. Jabs J, Devine C, Bisogni C et al. (2007) Trying to find the quickest way: employed mothers' constructions of time for food. J Nutr Educ Behav 39, 18-25.

12. Devine CM, Jastran M, Jabs J et al. (2006) 'A lot of sacrifices': work-family spillover and the food choice coping strategies of low-wage employed parents. Soc Sci Med 63, 2591-2603.

13. Gillman MW, Rifas-Shiman SL, Frazier AL et al. (2000) Family dinner and diet quality among older children and adolescents. Arch Fam Med 9240.

14. Taveras EM, Rifas-Shiman SL, Berkey CS et al. (2005) Family dinner and adolescent overweight. Obes Res 13, 900-906.

15. Chan JC \& Sobal J (2011) Family meals and body weight. Analysis of multiple family members in family units. Appetite 57, 517-524.
16. Sen B (2006) Frequency of family dinner and adolescent body weight status: evidence from the national longitudinal survey of youth, 1997. Obesity (Silver Spring) 14, 2266-2276.

17. Rollins BY, Belue RZ \& Francis LA (2010) The beneficial effect of family meals on obesity differs by race, sex, and household education: the national survey of children's health, 2003-2004. J Am Diet Assoc 110, 1335-1339.

18. Kramer RF, Coutinho AJ, Vaeth E et al. (2012) Healthier home food preparation methods and youth and caregiver psychosocial factors are associated with lower BMI in African American youth. J Nutr 142, 948-954.

19. Zick CD, Stevens RB \& Bryant WK (2011) Time use choices and healthy body weight: a multivariate analysis of data from the American Time Use Survey. Int J Behav Nutr Phys Act $\mathbf{8}, 84$.

20. Laska MN, Larson NI, Neumark-Sztainer D et al. (2012) Does involvement in food preparation track from adolescence to young adulthood and is it associated with better dietary quality? Findings from a 10 -year longitudinal study. Public Health Nutr 15, 1279-1287.

21. Smith LP, Ng SW \& Popkin BM (2013) Trends in US home food preparation and consumption: analysis of national nutrition surveys and time use studies from 1965-1966 to 2007-2008. Nutr J 12, 45.

22. Pabayo R, Spence JC, Cutumisu N et al. (2012) Sociodemographic, behavioural and environmental correlates of sweetened beverage consumption among pre-school children. Public Health Nutr 15, 1338-1346.

23. Boone-Heinonen J, Diez Roux AV, Kiefe CI et al. (2011) Neighborhood socioeconomic status predictors of physical activity through young to middle adulthood: the CARDIA study. Soc Sci Med 72, 641-649.

24. Centers for Disease Control and Prevention (2011) National Health and Nutrition Examination Survey. http://www.cdc. gov/nchs/nhanes.htm

25. Centers for Disease Control and Prevention (2007) Survey Questionnaires, Examination Components and Laboratory Components 2007-2008. Consumer Behavior Questionnaire. http://www.cdc.gov/nchs/nhanes/nhanes2007-2008/ questexam07_08.htm

26. Nielsen SJ, Siega-Riz AM \& Popkin BM (2002) Trends in energy intake in US between 1977 and 1996: similar shifts seen across age groups. Obes Res 10, 370-378.

27. Dubowitz T, Acevedo-Garcia D, Salkeld J et al. (2007) Lifecourse, immigrant status and acculturation in food purchasing and preparation among low-income mothers. Public Health Nutr 10, 396-404.

28. Devine CM, Wolfe WS, Frongillo EA Jr et al. (1999) Lifecourse events and experiences: association with fruit and vegetable consumption in 3 ethnic groups. J Am Diet Assoc 99, 309-314.

29. An R \& Sturm R (2012) School and residential neighborhood food environment and diet among California youth. Am J Prev Med 42, 129-135.

30. Lee H (2012) The role of local food availability in explaining obesity risk among young school-aged children. Soc Sci Med 74, 1193-1203.

31. Boone-Heinonen J, Gordon-Larsen P, Kiefe CI et al. (2011) Fast food restaurants and food stores: longitudinal associations with diet in young to middle-aged adults: the CARDIA study. Arch Intern Med 171, 1162-1170.

32. Oude Luttikhuis H, Baur L, Jansen $\mathrm{H}$ et al. (2009) Interventions for treating obesity in children. Cochrane Database Syst Rev issue 1, CD001872. 